\title{
Two Proofs of the ${ }_{8} \Psi_{6}$ Summation Theorem
}

\author{
By L. J. Slater and A. Lakin \\ (Received 19th December, 1951.)
}

1. Introduction. The ${ }_{6} \Psi_{6}$ summation theorem was first proved by Bailey ${ }^{1}$, who deduced it indirectly from a transformation of a well-poised ${ }_{8} \Phi_{7}$ series into two ${ }_{4} \Phi_{3}$ series. No direct proof of the theorem has been published, and, since it has interesting applications in the proofs of various identities which occur in combinatory analysis, for example the $A$ series of Rogers ${ }^{2}$ and some elegant identities due to Ramanujan ${ }^{3}$, we give two new proofs of the theorem in this paper.

The first proof (due to Slater) introduces a basic analogue of the Barnes type integral. The second (due to Lakin) is the basic analogue of an operational method used elsewhere 4 , and provides an application of Carlson's theorem.

2. First Proof. The notation is that introduced by Bailey, with the addition that

$$
\stackrel{N}{\Pi}\left(\begin{array}{c}
a \\
b ;
\end{array}\right)=\prod_{n=0}^{N} \frac{\left(1-a q^{n}\right)}{\left(1-b q^{n}\right)},
$$

and $\Pi$ is written for $\prod^{\infty}$. Thus,

$$
\Pi\left(\begin{array}{c}
a ; \\
b ;
\end{array}\right)=\prod_{n=0}^{\infty} \frac{\left(1-a q^{n}\right)}{\left(1-b q^{n}\right)} .
$$

Consider the integral

$$
I_{N}=\frac{1}{2 \pi i} \int P_{N}(s) d s
$$

where

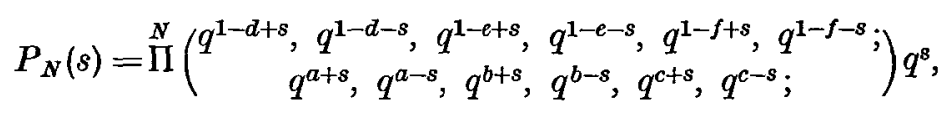

1 Bailey [1], §4.

2 See Slater [5], for full references.

3 Bailey [3].

- Burchnall [4]. 
and $q=e^{-t}, t>0$, taken round the contour

$$
A(-2 N-i \pi / t) \quad B(-2 N+i \pi / t) \quad C(2 N+i \pi / t) \quad D(2 N-i \pi / t),
$$

and assume that none of the members of the sequences

$$
q^{a \pm n}, q^{b \pm n}, q^{c \pm n}
$$

coincide or fall on the contour. By the periodicity of the integrand,

$$
\int_{B C}+\int_{D A}=0
$$

Also

$$
\int_{C D}=\frac{1}{2 \pi i} \int_{0}^{\pi / t}\left[P_{N}(2 N-i r)-P_{N}(2 N+i r)\right] d r
$$

and

$$
\int_{A B}=\frac{1}{2 \pi i} \int_{0}^{\pi / t}\left[P_{N}(-2 N-i r)-P_{N}(-2 N+i r)\right] d r .
$$

Both these integrals tend to zero as $N \rightarrow \infty$, provided

$$
\operatorname{Rl}(5-a-b-c-d-e)>0 .
$$

Thus we can equate to zero the sum of the residues at the poles of $P_{N}(s)$ in the $s$-plane. Now $1 / \Pi\left(q^{a+s}\right.$;) has poles within $A B C D$ at

$$
s=-a-n+2 \pi i k / t
$$

for some integer $k$. Hence $P_{N}(s)$ has increasing sequences of poles at $s=a+n, b+n, c+n$, and decreasing sequences of poles at

$$
s=-a-n,-b-n,-c-n, \text { for } n=0,1,2, \ldots
$$

Combining the residues at $s=a+n$ and $s=-a-n$, and using the symmetry in the integrand, we have

$$
\begin{array}{r}
\sum_{n=0}^{\infty} \Pi\left(\begin{array}{r}
q^{1+a-d+n}, q^{1-a-d-n}, q^{1+a-e+n}, q^{1-a-e-n}, q^{1+a-f+n}, q^{1-a-f-n} ; \\
q^{2 a+n}, q, q^{b+a+n},
\end{array} q^{b-a-n}, q^{c+a+n}, q^{c-a-n} ;\right. \\
\\
\times \frac{\left(q^{a+n}-q^{-a-n}\right)}{\left(q^{-n}\right)_{n}}+\operatorname{idem}(a ; b, c)=0,
\end{array}
$$

where "idem $(a ; b)$ " means that the preceding expression is to be repeated with $b$ and $a$ interchanged. 
If we put $a$ for $q^{\alpha}$, and so on, this gives, in the more conventional notation,

$$
\begin{aligned}
& \frac{1}{a} \Pi\left(\begin{array}{c}
a q / d, q / a d, a q / e, q / a e, a q / f, q / a f ; \\
a^{2} q, a b, b / a, a c, c / a ;
\end{array}\right) \\
& \times_{8} \Phi_{7}\left[\begin{array}{c}
a^{2}, a q,-a q, a b, a c, a d, a e, a f ; \\
a,-a, a q / b, a q / c, a q / d, a q / e, a q / f ; \overrightarrow{a b c d e f}
\end{array}\right] \\
& \text { +idem }(a ; b, c)=0 \text {, }
\end{aligned}
$$

where the restriction that $q$ is real can now be removed. This is the basic analogue of a result due to Whipple ${ }^{1}$. In (1) put $c=q / a$. The first and third series combine to give

$$
\begin{aligned}
& \frac{1}{a} \Pi\left[\begin{array}{c}
a q / d, a q / e, a q / f, q / a d, q / a e, q / a f ; \\
a^{2} q, a b, q, b / a, q / a^{2} ;
\end{array}\right]
\end{aligned}
$$

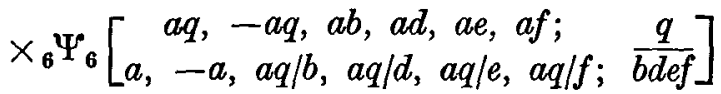

and the second series reduces to

$$
{ }_{6} \Phi_{5}\left[\begin{array}{l}
b^{2}, b q,-b q, b d, b e, b f ; \\
b,-b, b q / d, b q / e, b q / f ; b d e f
\end{array}\right]=\Pi\left(\begin{array}{c}
b^{2} q, q / d e, q / e f, q / d f ; \\
b q / d, b q / e, b q / f, q / b d e f ;
\end{array}\right)
$$

Hence, after a little reduction, we have the required result,

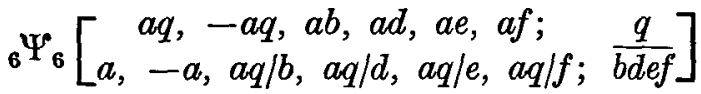

$$
\begin{aligned}
& =\Pi\left[\begin{array}{c}
a^{2} q, q / b d, q / b e, q / b f, q / d e, q / d f, q / e f, q, q / a^{2} ; \\
q / a b, q / a d, q / a e, q / a f, a q / b, a q / d, a q / e, a q / f, q / b d e f ;
\end{array}\right] .
\end{aligned}
$$

3. Second Proof. Let

$$
\Psi={ }_{4} \Psi_{4}\left[\begin{array}{c}
a b, a c, a d, a e ; \\
a q / b, a q / c, a q / d, a q / e ;
\end{array}\right]
$$

and let $Q$ be the operator $q^{x d / d x}$ with the property

$$
Q f(x)=f(q x)
$$

1 Bailey [1], $\S(4.6)$. 
where $f(x)$ is a polynomial or power series in $x$. Then

$$
\begin{aligned}
\left(1-\frac{a}{b} Q\right)(1-a b Q) \Psi=\left(1-\frac{a}{b}\right)(1-a b) & \\
& \times{ }_{4} \Psi_{4}\left[\begin{array}{c}
a b q, a c, a d, a e ; \\
a / b, a q / c, a q / d, a q / e ;
\end{array}\right],
\end{aligned}
$$

the effect of the operator being to multiply $b$ by $q$ whenever it occurs in the series, which remains well-poised. Further,

$$
\left(1-a^{2} Q^{2}\right) \Psi=\left(1-a^{2}\right)_{6} \Psi_{6}\left[\begin{array}{c}
a q,-a q, a b, a c, a d, a e ; \\
a,-a, a q / b, a q / c, a q / d, a q / e ;
\end{array}\right]
$$

which introduces the first and second parameters of special form.

The $q$-difference equation satisfied by $\Psi$ is

$$
\begin{aligned}
& {\left[\left(1-\frac{a}{b} Q\right)\left(1-\frac{a}{c} Q\right)\right.}\left(1-\frac{a}{d} Q\right)\left(1-\frac{a}{e} Q\right) \\
&-x(1-a b Q)(1-a c Q)(1-a d Q)(1-a e Q)] \Psi=0 .
\end{aligned}
$$

The operator in (5) may be written

$$
\begin{aligned}
{\left[\left(1-\sigma_{-1} a Q+\sigma_{-2} a^{2} Q^{2}-\sigma_{-3} a^{3} Q^{3}+\sigma_{-4} a^{4} Q^{4}\right)\right.} \\
\\
\left.-x\left(1-\sigma_{1} a Q+\sigma_{2} a^{2} Q^{2}-\sigma_{3} a^{3} Q^{3}+\sigma_{4} a^{4} Q^{4}\right)\right],
\end{aligned}
$$

where $\sigma_{r}$ is the $r$-th elementary symmetric function of the four parameters $b, c, d$ and $e$. Put $x=1 / b c d e=\sigma_{-4} ;$ then since $\sigma_{1} \sigma_{-4}=\sigma_{-3}$, etc., the operator may be written

$$
\left[\left(1-\sigma_{-4}\right)\left(1-a^{4} Q^{4}\right)-\left(\sigma_{-1}-\sigma_{-3}\right) a Q\left(1-a^{2} Q^{2}\right)\right]
$$

or

$$
\left[B(1-a b Q)\left(1-\frac{a}{b} Q\right)+C(1-a c Q)\left(1-\frac{a}{c} Q\right)\right]\left(1-a^{2} Q^{2}\right),
$$

where $B$ and $C$ are undetermined constants. These may be evaluated by putting $Q=1 / a c, 1 / a b$ in turn in (5) and (6), whence we find

$$
B=\frac{(1-1 / c d)(1-1 / c e)}{1-b / c}, \quad \text { and } C=\frac{(1-1 / b d)(1-1 / b e)}{1-c / b} .
$$


Using (3) and (4) to perform on $\Psi$ the operation indicated by (6), we have

$$
\begin{aligned}
& \frac{(1-1 / c d)(1-1 / c e)(1-a b)(1-a / b)}{1-b / c} \Psi_{6}\left[\begin{array}{c}
a q,-a q, a b q, a c, a d, a e ; \\
a,-a, a / b, a q / c, a q / d, a q / e ; b c d e
\end{array}\right] \\
& +\frac{(1-1 / b d)(1-1 / b e)(1-a c)(1-a / c)}{1-c / b} \\
& \times{ }_{6} \Psi_{6}\left[\begin{array}{c}
a q,-a q, a b, a c q, a d, a e ; \\
a,-a, a q / b, a / c, a q / d, a q / e ;
\end{array} \frac{1}{b c d e}\right]=0 .
\end{aligned}
$$

If we arrange this and write $b / q$ for $b$, then

$$
\begin{aligned}
& \Psi(b, c) \equiv{ }_{6} \Psi_{8}\left[\begin{array}{c}
a q,-a q, a b, a c, a d, a e ; \\
a,-a, a q / b, a q / c, a q / d, a q / e ; \overrightarrow{b c d e}
\end{array}\right] \\
& =\frac{(1-q / b d)(1-q / b e)(1-1 / a c)(1-a / c)}{(1-1 / c d)(1-1 / c e)(1-q / a b)(1-a q / b)} \Psi(b / q, c q),
\end{aligned}
$$

or, on applying the transformation $N$ times,

$$
\Psi(b, c)=\Pi^{N-1}\left(\begin{array}{c}
q / b d, q / b e, 1 / a c, a / c ; \\
1 / c d, 1 / c e, q / a b, a q / b ;
\end{array}\right) \Psi\left(b q^{-N}, c q^{N}\right) .
$$

This equation is a two-term difference relation satisfied by the series. Such a relation must exist in order that a hypergeometric series should be summable. To show that (7) is still true for non-integral values of $N$ we apply Carlson's theorem to the function

$$
\begin{aligned}
& f(z)=\Pi\left(q^{1+z} / b d, q^{1+z} / b e, q / a c, a q / c, q^{1-z} / c d, q^{1-z} / c e, q / a b, a q / b ;\right) \Psi(b, c) \\
& \text { - }\left(q / b d, q / b e, q^{1-z} / a c, a q^{1-z} / c, q / c d, q / c e, q^{1+z} / a b, a q^{1+z} / b ;\right) \\
& \times \Psi\left(b q^{-z}, c q^{z}\right)
\end{aligned}
$$

which is, in effect, (7) multiplied by a suitable factor, with $N$ replaced by $z$.

It is easy to establish by the usual arguments ${ }^{1}$ that for $\operatorname{Rl}(z) \geqslant 0$ this function is regular and of the required order for large values of $z$, subject to certain restrictions on the parameters which may be removed from the final result. By $(7), f(z)=0$ if $z=0,1,2, \ldots$, and therefore by Carlson's theorem it is identically zero. In particular it is zero if $q^{z}=b / a$, when $\Psi\left(b q^{-z}, c q^{2}\right)$ reduces to a summable ${ }_{6} \Phi_{5}$ and the required result (2) follows immediately.

1 Bailey [2], (5 . 3) and (5.4). 
The operator (6) may be written in another form, thus:

$$
\begin{array}{r}
{\left[A(1-a b Q)\left(1-\frac{a}{b} Q\right)+B(1-a \lambda Q)\left(1-\frac{a}{\lambda} Q\right)\right]\left(1-a^{2} Q^{2}\right)} \\
\equiv\left[\left(1-\frac{a}{b} Q\right)\left(1-\frac{a}{c} Q\right)\left(1-\frac{a}{d} Q\right)\left(1-\frac{a}{e} Q\right)\right. \\
\left.-\frac{1}{b c d e}(1-a b Q)(1-a c Q)(1-a d Q)(1-a e Q)\right]
\end{array}
$$

where $\lambda$ is dependent on $a, b, c, d$ and $e$.

Using the operator in this form, we obtain

$$
\begin{aligned}
& A(1-a b)\left(1-\frac{a}{b}\right){ }_{6} \Psi_{6}\left[\begin{array}{c}
a q,-a q, a b q, a c, a d, a e ; \\
a,-a, a / b, a q / c, a q / d, a q / e ; b c d e
\end{array}\right] \\
& +B(1-a \lambda)(1-a / \lambda) \\
& \times_{8} \Psi_{8}\left[\begin{array}{c}
a q,-a q, a \lambda q, a q / \lambda, a b, a c, a d, a e ; \\
a,-a, a / \lambda, a \lambda, a q / b, a q / c, a q / d, a q / e ; b c d e
\end{array}\right]=0,
\end{aligned}
$$

where $A$ and $B$ are constants which can be determined. The ${ }_{6} \Psi_{6}$ is summable, and so therefore is the ${ }_{8} \Psi_{8}$. It is interesting to notice the existence of this summable ${ }_{8} \Psi_{8}$, though there is little point in stating the result in detail.

\section{REFERENCES.}

[1] W. N. Bailey, "Series of hypergeometric type which are infinite in both directions", Quart. J. of Math. (Oxford), 7 (1936), 105-115.

[2] _-_ Generalised Hypergeometric Series (Cambridge, 1935).

[3] —_ "A note on two of Ramanujan's formulae", Quart. J. of Math. (Oxford) (2), 3 (1952), 29-31.

[4] J. L. Burchnall and A. Lakin, "The theorems of Saalschütz and Dougall ", Quart. J. of Math. (Oxford) (2), 1 (1950), 161-164.

[5] L. J. Slater, "Further identities of the Rogers-Ramanujan type", Proc. London Math. Soc. (2), 54 (1951), 147-167.

UNIVERSity Mathematical LaboRatoRy, Cambridge.
College of Technology, Manchester. 